direct cost of care for these patients exceeded $\$ 23$ million.

Since this investigation ended (August 1, 1993), there were 82 additional RFLP-confirmed patients that met the case definition; the outbreak began with 15 RFLP-confirmed cases in 1990, peaked with 122 cases in 1992, and decreased to 19 cases in 1995.

FROM: Frieden TR, Sherman LF, Maw KL. A multiinstitutional outbreak of highly drug-resistant tuberculosis. JAMA 1996;276:1229-1235.

\section{Routine Cultures of Environment for Legionella?}

Ever since Legionella pneumophila was isolated and characterized and typing systems became available, there has been a seemingly endless debate between those who promote the strategy of routinely monitoring hospital water for the microorganism and those (notably Centers for Disease Control and Prevention) who maintain that such monitoring is basically a waste of resources, because all hospital water is colonized with L pneumophila, and disease surveillance should be the prime priority.

Researchers at the VA Medical Center and the University of Pittsburgh School of Medicine in Pittsburgh, Pennsylvania, reported on a study that supports the case for routine environmental cultures of hospital water. Surveillance for Legionnaires' disease in hospitalized patients with fever and pulmonary infiltrates was done for 12 months. For every patient with nosocomial pneumonia, additional tests for legionellae were performed for urinary antigen, sputum culture, and serology. Cultures of the environment for legionellae was done in patient-care areas and the hot-water storage tanks of the hospital. Pulsed-field gel electrophoresis (PFGE) was used as a typing system to determine concordance between patient and environmental isolates.

Of 102 patients identified during the study period, 3 had nosocomial pneumonia caused by $L$ pneumophila Serogroup 5. This serogroup was recovered from 4 of 5 hotwater storage tanks $(10-1,000 \mathrm{CFU} / \mathrm{mL})$. Furthermore, the hospital water supply was colonized with $L$ pneumophila Serogroup 5, as shown by studies conducted over a 10 -year period; isolates were available from 1984, 1986, and 1994. No other serotypes were isolated. The Serogroup Type 5 isolates from the three infected patients had the same PFGE pattern as the Serogroup 5 isolates from the water supply. In contrast, 12 L pneumophila serogroup isolates from eight other institutions had different PFGE patterns. The authors conclude that routinely obtaining cultures for legionellae from the environment may be important in stimulating the application of laboratory testing for Legionella, which can identify unsuspected patients with nosocomial Legionnaires' disease.

FROM: Chang FY, Jacobs SL, Colodny SM, Stout JE, $\mathrm{Yu}$ VL. Nosocomial Legionnaires' disease caused by Legionella pneumophila Serogroup 5: laboratory and epidemiologic implications. J Infect Dis 1996;174:1116-1119.

\section{New Finding on Biofilm and Coagulase-Negative Staphylococci}

Coagulase-negative staphylococci, for the most part Staphylococcus epidermidis, are the most frequent organisms responsible for infections of implanted medical devices. Strains of these organisms have been shown in the laboratory to produce a macroscopically visible, adherent biofilm on test tubes or plates, and the biofilm production occurs in two phases. First, there is rapid primary attachment of $S$ epidermidis cells to a surface, followed by accumulation of cells in multilayered cell clusters. The latter phase requires intracellular adhesion, and a specific polysaccharide has been described: polysaccharide intercellular adhesin (PIA), which is different from the many other polysaccharides produced by the organism.

Researchers at the Institute for Medicine, Microbiology, and Immunology at the University of Eppendorf, Hamburg, Germany, studied the association of biofilm production with expression of PIA in 179 isolates of S epidermidis.

They found that there was a significant positive association between biofilm production and PIA expression: $86.8 \%$ of biofilm-producing strains produced PIA. In contrast, $88.6 \%$ of biofilm negative strains did not express PIA.

The authors conclude that PIA is an important factor involved in biofilm accumulation of the majority of $S$ epidermidis clinical isolates and that studies to determine clinical relevance are needed.

FROM: Mack D, Haeder M, Siemssen N, Laufs R. Association of biofilm production of coagulase-negative staphylococci with expression of a specific polysaccharide intercellular adhesin. J Infect Dis 1996;174:881-884.

\section{Restrict Antibiotics, Control VRE}

Vancomycin-resistant enterococci (VRE) have evolved to the point where they are major nosocomial pathogens. They can be the infection control practitioners' worst nightmare when increasingly found in the gut of patients and the CDC's recommendations are not working. Such is the plight of investigators from the Brooklyn VA Center, who describe the battle with VRE that began in the fall of 1991. Cultures of nosocomial VRE were increasing, as were the number of patients who were colonized with VRE. In spring 1993, a number of infection control steps were initiated at the hospital: VRE patients were placed in private rooms in isolation; the inguinal and perineal areas of infected patients were washed with chlorhexidine; gloves were required by staff, and they also used chlorhexidine soap for handwashing; electronic thermometers were removed; and an infection control clinician made frequent rounds to reinforce adherence to these measures. In addition, a 1:100 dilution of household bleach was used to clean environmental surfaces.

When a point prevalence survey performed in January 1995 showed that there was still widespread gastrointestinal colonization with VRE, a second intervention 
was initiated, and this was a policy that required an infectious diseases physician to approve the use of cefotaxime (the major cephalosporin used at the hospital). Vancomycin use also required the same type of approval, and the use of clindamycin also was restricted because of an outbreak of Clostridium difficile colitis that was occurring at the same time.

After 6 months, the average monthly use of cefotaxime, ceftazidime, vancomycin, and clindamycin had decreased by $84 \%, 55 \%, 34 \%$, and $80 \%$, respectively; the point prevalence of fecal colonization with VRE decreased from $47 \%$ to $15 \%$, and the number of patients whose clinical specimens were culture-positive for VRE also decreased gradually. The authors concluded that the change in antibiotic use appeared to have affected the VRE outbreak when previous measures had failed.

FROM: Quale J, Landman D, Guillermo S, et al. Manipulation of a hospital antimicrobial formulary to control an outbreak of vancomycin-resistant enterococci. Clin Infect Dis 1996;23:1020-1025.

\section{Bacterial Contamination of Dental Unit Water Lines}

In Canada, the current public health standards consider water to be safe for consumption when it contains a total bacterial count of less than $500 \mathrm{CFU} / \mathrm{mL}$ and is free of coliform bacteria. However, as most water microbiologists have known for years, the gram-negative water bacteria can grow quite well in water, and, in doing so, form biofilms and thus colonize water distribution pipes. This ecosystem offers a never-ending supply of gram-negative bacteria in the water. For the most part, ingestion of water containing this group of bacteria does not cause disease in humans. However, the introduction of these bacteria into open lesions, such as one experiences in a dental unit, has caused concern. Researchers from the dental school at the University of Montreal, Quebec, Canada, conducted a study that was designed to observe bacterial colonization of dental unit water lines from a dynamic standpoint through multilevel analysis and species identification, distribution, and fluctuation in number over time. Water samples were collected from 123 dental units at the dental school, and 30 of the units were included in a multilevel analysis that consisted of samples taken at the beginning of the workday and after a 2-minute purge, and quantitative assays were performed and isolates identified.

Among the results were: the highest colony counts were achieved using R2Am medium (a reduced nutrient medium) at $22^{\circ} \mathrm{C}$, as compared to TSA and blood agar at $37^{\circ} \mathrm{C}$; the colony counts reached a plateau after 7 days. At the beginning of the workday, the air-water syringes and highspeed water lines contained water with counts over 100,000 $\mathrm{CFU} / \mathrm{mL}$, and, after a 2-minute purge, the counts were reduced by approximately 2 logs. The predominant bacterial species recovered were characteristic gram-negative water bacteria, such as Sphyngomonas paucimobilis, Acinetobacter calcoaceticus, Methylobacter mesophilicum, and Pseudomonas aeruginosa. Dental units contaminated by $P$ aeruginosa had the highest total bacterial counts; $P$ aeruginosa never was isolated from the tap water feeding the dental units.

The authors concluded that dental unit water lines should be considered an aquatic ecosystem in which opportunistic pathogens successfully colonize the water pipes, increasing the concentration of bacteria to potentially dangerous levels Although the clinical significance is yet to be documented, a number of techniques for purging or disinfecting the systems are suggested.

FROM: Barbeau J, Tanguay R, Faucher E, et al. Multiparametric analysis of waterline contamination in dental units. Appl Environ Microbiol 1996;62:3954-3959.

\section{APHA Addresses Contaminated Dental Unit Water Lines}

Bacterial contamination of dental unit water lines has been a continuing subject that has been discussed by the Microbial Contamination Control Committee of the American Public Health Association's (APHA) Laboratory Section. The committee met during the meetings of the APHA, November 17-21, in New York City. The committee has a keen and ongoing interest in microbial contamination associated with medical devices that are difficult to clean and thus renders such devices difficult to sterilize or disinfect. In the case of dental unit water lines, the committee has drafted a policy statement for endorsement by the APHA to encourage the dental community to realize that dental unit water lines can be contaminated excessively with gram-negative water bacteria. Because many of these can be potential pathogens, the committee urges that purging and disinfection procedures be done routinely. 